\title{
UN NUEVO ENCUENTRO CON EL RECURRENTE PROBLEMA DE BASILEA: $\sum_{K=1}^{\infty} \frac{1}{K^{2}}$
}

A NEW ENCOUNTER WITH THE RECURRING BASEL PROBLEM: $\sum_{K=1}^{\infty} \frac{1}{K^{2}}$

\author{
Harold VACCA1, Lucio ROJAS², Nicolás CONDE \\ 1 Universidad Distrital Francisco José de Caldas, Facultad Tecnológica, Bogotá, Colombia. \\ 2 Universidad Militar Nueva Granada, Departamento de Matemáticas, Bogotá, Colombia. \\ *Autor corresponsal. E-mail: lucio.rojas@unimilitar.edu.co.
}

Historia del artículo
Recibido: Enero 19, 2018
Evaluado: Abril 19, 2018
Aceptado: Mayo 11, 2018
Disponible: Mayo 31, 2018

\section{Resumen |}

Los espacios vectoriales se han considerado una herramienta fundamental para las aplicaciones modernas del álgebra lineal; en este sentido, las funciones (señales) se consideran vectores. Cuando el espacio vectorial se dota con un producto interior, la proyección de una función sobre un subespacio es análoga al cálculo de una aproximación a un vector del espacio, por lo general, de dimensión finita, mediante un número finito de funciones de una base de tipo ortogonal. En el presente artículo se observan los antecedentes y las soluciones al problema de Basilea (PB) o $\sum_{K=1}^{\infty} \frac{1}{K^{2}}$, desde su origen a mediados del siglo XVII hasta hoy, es decir, 372 años de un problema que aparece de forma recurrente en el ambiente matemático. Se presenta como novedad una solución por medio de la teoría de los espacios vectoriales, en concreto, mediante la utilización de proyecciones de una función sobre un subespacio; se exhiben los resultados de aplicar la solución en el cálculo de $\pi$ y su comparación con fracciones que lo han representado históricamente.

Palabras clave: espacios vectoriales; espacio de Hilbert; proyecciones; base ortogonal; problema de Basilea; Euler

Abstract |

Vector Spaces have been considered a basic tool for modern applications of Linear Algebra; in this sense, the functions -signals- are considered as vectors. When a Vector Space is endowed with an inner product, the projection of a function on a subspace is analogous to the calculation of an approach to a vector of the space, usually finite dimensional, by a finite number of functions of a base of orthogonal type. In this article, will take place a look of the background and solutions to the Basel problem (BP) or $\sum_{K=1}^{\infty} \frac{1}{K^{2}}$, since its inception in the mid-seventeenth century to today, i.e.: 372 years of a problem that recurring appears in the mathematical environment. It's presented as a novelty a solution through the theory of vector spaces, specifically using projections of a function on a subspace. It exhibits the results of applying the solution to calculating of $\pi$ and comparison with fractions that historically have represented him.

Keywords: vector spaces; hilbert space; projections; orthogonal basis; basel problem; Euler

\section{INTRODUCCIÓN}

Los siglos XVII y XVIII fueron de importancia capital para la matemática que se conoce en la actualidad, porque fue entonces cuando se plantearon y desarrollaron problemas que cambiaron la perspectiva del mundo matemático que hoy conocemos.
Particularmente en Basilea, la ciudad natal de Leonhard Euler (1707-1783), se constituyó un epicentro de tales ideas, ya que, además, fue el lugar donde vivió, desde 1622, una de las familias de matemáticos más notables de la época: los Bernoulli; es decir, puede considerarse una ciudad suiza de pergaminos (Aigner y Ziegler, 2004). 
Allí, a comienzos del siglo XVIII, las series infinitas fueron el principal tema de investigación en matemáticas. Analizar el comportamiento de estas e intentar demostrar su convergencia sería uno de los principales retos. Si un matemático resolvía alguno, gozaría entonces de un reconocimiento proporcional a la dificultad de este.

Entre los significativos se nominó uno como "el de Basilea", planteado por primera vez hacia 1644 en Novae Quadrature Aritmeticae por Pietro Mengoli (1625-1686), matemático italiano de la época. En el primero de los tres capítulos que la conforman, Mengoli logró demostrar y calcular el valor de la ecuación (1) (Aigner y Ziegler, 2004):

$$
\sum_{n=1}^{\infty} \frac{1}{n(n+1)}(1)
$$

En los otros dos se centró en demostrar las series de término general:

$$
\sum_{n=1}^{\infty} \frac{1}{n(n+r)} r \in N_{(2)}
$$

Encontró los resultados de (2) para cualquier $r$ entero positivo, pero no para $r=0$.

La ecuación (2) para $r=0$ se convirtió entonces en el conocido problema de Basilea (PB). Presentado a Gottfried Leibniz (1646-1716) por Henry Oldenburg, teólogo y filósofo alemán secretario de la Royal Society hacia 1673 , el padre del cálculo infinitesimal intentó encontrar el valor al que presumiblemente convergía.

$$
\sum_{n=1}^{\infty} \frac{1}{n}=1+\frac{1}{2}+\frac{1}{3}+\frac{1}{4}+\frac{1}{5}+\cdots
$$

Después de haber encontrado que la serie armónica (3) divergía, finalmente se rindió. Luego de estas frustraciones, Jacob Bernoulli, obsesionado logró demostrar en Positiones Aritmeticae de Seriebus Infinitus Earumque Summa Finita el resultado de la suma de las series con forma:

$$
\sum_{n=1}^{\infty} \frac{1}{n^{2}-k^{2}} ; k \in N(4)
$$

En particular, estableció que (4) con k = o convergía, pero no pudo hallar el resultado de la suma.

En adelante muchos fueron los matemáticos que intentaron solventar el PB y fracasaron en el intento: el inglés Jhon WaIlis (1616-1703) en Arithmetica Infinitorum (1655) aproximó el valor de dicha serie a 1.645 (hoy se sabe que cometió un error menor a una milésima, pues se necesitan 1.071 términos para dar una precisión de tres cifras decimales). Hacia 1721, en una carta a su hijo Daniel, Johann Bernoulli especifica que el resultado de esta serie se halla en torno al valor de $\frac{8}{5}$; en 1729, el matemático prusiano Christian Goldbach (1690-1764) acotó el valor entre $\frac{41}{35}$ $\mathrm{y} \frac{5}{3}$; en 1730 el matemático escocés James Stirling (1692-1770) en Methodus Differentialis encontró el valor de 1.644934066 , resultado correcto hasta la novena cifra decimal.

A lo largo de este documento se considera un espacio vectorial $\mathrm{H}$ finito dimensional equipado con un producto interior', con la norma asociada $\|\|=.(\langle, 1 / 2)$; una base ortogonal formada por vectores $v_{k}$ (susceptible de normalizarse) para $\mathrm{H}$, donde cada elemento $\mathrm{f}$ de $\mathrm{H}$ tiene una representación:

$$
f=\sum_{K=1}^{\infty}\left\langle f, v_{k}\right\rangle \cdot v k
$$

A fin de realizar una extensión de estos conceptos a espacios vectoriales infinito-dimensionales se recurre a los espacios de Hilbert $\left(\mathrm{H}=\mathrm{L}_{2}[-\pi, \pi]\right.$ de las funciones cuadrado integrable en $[-\pi, \pi])$. Estos espacios tienen la propiedad de que cada sucesión de Cauchy en $\mathrm{H}$ converge en $\mathrm{H}$ (Kolman y Hill, 2014). En razón a lo anterior, dada una base ortogonal infinito-dimensional para el espacio $\mathrm{H}$, cada $\mathrm{f}$ puede expresarse como:

$$
f=\sum_{K=1}^{\infty}\left\langle f, v_{k}\right\rangle \cdot v k
$$

De manera que:

$$
\left\|f-\sum_{\boldsymbol{K}=1}^{\infty}\left\langle f, v_{k}\right\rangle \cdot v k\right\| \rightarrow 0_{\text {Cuando } n \rightarrow \infty}
$$

\section{MATERIALES Y MÉTODOS |}

Inicialmente, se realiza una documentación sobre los métodos implementados por los matemáticos que encontraron la solución al PB a través de diversas técnicas de aproximación. Posteriormente, se considera la proyección ortogonal de f sobre un subespacio $\mathrm{W}$ como método alternativo para solucionar el PB. Luego, se considera en perspectiva el PB relacionado con la transformación integral de Mellin. Como validación de la solución alternativa se compara con cálculos sobre $\pi$. Finalmente, se establecen las conclusiones.

\section{Euler y sus aproximaciones}

En 1731, Euler logra calcular el valor de la serie de forma correcta para los primeros 20 decimales (Dunham, 2004):

$$
1+\frac{1}{2^{2}}+\frac{1}{3^{2}}+\cdots \frac{1}{1000^{2}}=1,643934 \ldots . \text { (5) }
$$

Para obtener este resultado, Euler se basó en la integral (Euler, 1748):

$$
\int_{0}^{\frac{1}{2}}-\frac{\ln (1-t)}{t} d t
$$


Obteniendo que:

$$
\sum_{n=1}^{\infty} \frac{1}{n^{2}}=\log ^{2}(2)+\sum_{k=1}^{\infty} \frac{1}{k^{2} 2^{k-1}}
$$

Dado que Euler conocía el valor de log(2) para algunos decimales, pudo dar una aproximación más exacta de la serie. No obstante, después de darla, no se sintió satisfecho, pues no encontró el valor exacto al cual la serie convergía. Hacia 1734 sucedió lo que todos los matemáticos de la época esperaban con ansias: Euler anuncia la demostración del resultado definitivo.

Para esto, parte de la función y su expansión en polinomios de Taylor (Knopp, 1996):

$$
\operatorname{Sen} x=x-\frac{x^{3}}{3 !}+\frac{x^{5}}{5 !}-\cdots
$$

Ignorando la convergencia uniforme, divide (8) entre x:

$$
\frac{\operatorname{Sen} x}{x}=1-\frac{x^{2}}{3 !}+\frac{x^{4}}{5 !}-\cdots
$$

Supone luego $\frac{\operatorname{Sen} x}{x}$ como un producto infinito, y como conocía previamente que la función Sen $x$ se anula en $x=n \pi$ con $n \in N$, expresa (9) como:

$$
\frac{\operatorname{Sen} x}{x}=\left(1-\frac{x}{\pi}\right)\left(1+\frac{x}{\pi}\right)\left(1-\frac{x}{2 \pi}\right)\left(1+\frac{x}{2 \pi}\right) \ldots
$$

Simplificando:

$$
\frac{\operatorname{Sen} x}{x}=\left(1-\frac{x^{2}}{\pi^{2}}\right)\left(1-\frac{x^{2}}{4 \pi^{2}}\right) \ldots
$$

Operando algebraicamente (11):

$$
-\left(\frac{1}{\pi^{2}}+\frac{1}{4 \pi^{2}}+\cdots\right) x^{2}=-\frac{1}{\pi^{2}}\left(\sum_{n=1}^{\infty} \frac{1}{n^{2}}\right) x^{2}(12)
$$

Este término debe coincidir con el coeficiente de $x^{2}$ de la expansión de Taylor que es -1/3!:

$$
\frac{1}{3 !}=\frac{1}{\pi^{2}} \sum_{n=1}^{\infty} \frac{1}{n^{2}}
$$

Igualando los coeficientes, Euler prueba el PB:

$$
\frac{\pi^{2}}{6}=\sum_{n=1}^{\infty} \frac{1}{n^{2}}
$$

El resultado, aunque válido, fue criticado por muchos -entre otros por Johann Bernoulli- porque se basó en las raíces de $\frac{\operatorname{Sen} x}{x}$ para escribirlo en forma de producto infinito.

Johann recomendó a Euler que demostrara la unicidad de tales raíces, pues existen funciones que tienen las mismas raí- ces, pero su expansión mediante series de Taylor es completamente distinta; por ejemplo:

$$
e^{x} \frac{\operatorname{Sen} x}{x}
$$

Dado que las críticas al método seguían, Euler lo que intentó fue demostrar que sus afirmaciones eran correctas por otras vías. Mientras esto ocurría, otra demostración distinta se publicó de forma anónima con el nombre de Demostration de la somme de cette suite $1+\frac{1}{4}+\frac{1}{9}+\frac{1}{16}+\cdots$, en una revista alemana; pero en 1844 fue redescubierta y adjudicada a Euler gracias a K. F. Gauss (1777-1855) (Dunham, 2004).

En esta, Euler comienza por considerar una circunferencia de radio 1, y s un ángulo; toma y por infinitesimales:

$$
d s=\frac{d x}{\sqrt{1-x^{2}}}
$$

E integrando:

$$
s=\int \frac{d x}{\sqrt{1-x^{2}}}
$$

Al multiplicar ambas expresiones término a término:

$$
s d s=\frac{d x}{\sqrt{1-x^{2}}} \int \frac{d x}{\sqrt{1-x^{2}}}
$$

Al "solucionar" dicha integral y aplicar el teorema del binomio generalizado, llega a la solución:

$$
\sum_{i m p a r} \frac{1}{n^{2}}=\frac{\pi^{2}}{8}
$$

En este punto, Euler razona lo siguiente "como todo número es resultado de una potencia de dos y un número impar, los cuadrados deben ser un cuadrado impar, multiplicado por una potencia de cuatro", por lo que multiplica (19) por la serie geométrica de razón $\frac{1}{4}$, llegando así a (14).

Años más tarde, hacia 1755, Euler publica en Institutiones Calculis Differentialis una solución a las series de la forma:

$$
\sum_{n=1}^{\infty} \frac{1}{n^{2 k}} ; k \in N(20)
$$

Relacionando:

$$
V(x)=\frac{x}{e^{x}-1}=\sum_{n=1}^{\infty} \frac{B_{n}}{n !} x^{n}
$$

Donde $B_{n}$ son los números de Bernoulli, los cuales aparecen en las sumas tipo, con k par:

$$
\sum_{n=1}^{\infty} \frac{1}{n^{k}} ; k \in N
$$




\section{Fourier y sus aproximaciones}

Fourier (1768-1830) aparece en este momento histórico del PB. Si se considera $f(x)=x$ en el intervalo $[0,1]$; desarrollando por series de Fourier donde $C_{n}$ coeficientes:

$$
c_{n}=\int_{0}^{1} x e^{-2 \pi i n x} d x=\frac{1}{2 \pi i n}(23)
$$

Se obtiene:

$$
\|f\|^{2}=\int_{0}^{1} x^{2} d x=\frac{1}{3}, c_{o}=\int_{0}^{1} x d x=\frac{1}{2}
$$

Por Parseval:

$$
\|f\|^{2}=\sum_{n=-\infty}^{\infty}\left|c_{n}\right|^{2}
$$

Se tiene:

$\|f\|^{2}=\frac{1}{3}=\sum_{n=-\infty}^{\infty}\left|c_{n}\right|^{2}=\frac{1}{4}+\sum_{n=-\infty}^{\infty} \frac{1}{4 \pi^{2} n^{2}} ; n \neq 0$

Es decir:

$$
\frac{1}{12}=\frac{2}{4 \pi^{2}} \sum_{n=1}^{\infty} \frac{1}{n^{2}}
$$

Operando, se obtiene (14) (Simmons, 2002).

\section{William Leveque y la solución del PB por integrales dobles}

En 1956, el matemático estadounidense William J. LeVeque (1923-2007), quien trabajó, fundamentalmente, la teoría de números, llegó a la solución de PB, al plantear la integral doble:

$$
I=\int_{0}^{1} \int_{0}^{1} \frac{1}{1-x y} d x d y
$$

Al evaluar $\frac{1}{1-x y}$ como solución a la serie geométrica de razón $(x y)^{n}$ y realizar una adecuada sustitución, LeVeque demuestra (14) (Pérez, 2007).

En Brendan (2013) se trata la misma solución, pero divulgada por el popular Tom Apostol (1923-2016), quien en 1983 la publicó en Mathematical Intelligencer.

Cabe anotar que hacia 1996 (Apostol, 1996) se rememoraba la formulación de Euler que relaciona las series aquí expuestas con el producto de números primos y que expresa la solución al PB:

$$
\sum_{k=1}^{\infty} \frac{1}{n^{s}}=\prod_{p} \frac{p^{s}}{p^{s}-1} \text { con p primo }
$$

Por tanto, tomando escribe:

$$
\frac{\pi^{2}}{6}=\frac{2^{2}}{2^{2}-1} \times \frac{3^{2}}{3^{2}-1} \times \frac{5^{2}}{5^{2}-1} \times \frac{7^{2}}{7^{2}-1} \times \ldots
$$

Los hermanos Yaglom y la solución trigonométrica del PB Para esto, parten de los valores de la función, para todo $m \geq 1$ :

$$
\begin{aligned}
\operatorname{Cot}^{2}\left(\frac{\pi}{2 m+1}\right)+ & \operatorname{Cot}^{2}\left(\frac{2 \pi}{2 m+1}\right) \\
+ & \cdots \operatorname{Cot}^{2}\left(\frac{m \pi}{2 m+1}\right) \\
& =\frac{2 m(2 m-1)}{6}
\end{aligned}
$$

Utilizando técnicas de variable compleja y una desigualdad obtenida del teorema del emparedado, logran demostrar que dicha función converge a $\frac{\pi^{2}}{6}$ cuando $\mathrm{m} \rightarrow \infty$ y llegan así a la solución del PB (Pérez, 2007).

\section{Solución por sumas telescópicas}

Una demostración realizada por Daniel Daners, en la Universidad de Sídney en el 2006, parte de la idea de escribir las sumas parciales de la serie como una suma telescópica y estimar el término de error.

La prueba se basa en las relaciones de recurrencia entre las integrales obtenidas al integrar por partes y realizar estimaciones simples.

Las integrales utilizadas son:

$$
A_{n}=\int_{0}^{\pi / 2} \operatorname{Cos}^{2 n} x d x \text { y } B_{n}=\int_{0}^{\pi / 2} x^{2} \operatorname{Cos}^{2 n} x d x
$$

Para $n \geq 0$, una forma de expresar la serie en forma de suma telescópica es:

$$
0 \leq \frac{\pi^{2}}{6}-\sum_{k=1}^{\infty} \frac{1}{k^{2}}=2 \frac{B_{n}}{A_{n}} \leq \frac{\pi^{2}}{4(n+1)}
$$

Del resultado anterior y utilizando identidades trigonométricas, se obtiene:

$$
\sum_{k=1}^{n-1} \frac{1}{k^{2}}=\frac{\pi^{2}}{6}-\frac{\pi}{4} \frac{(2 n) ! !}{(2 n-1) ! !} B_{n}
$$

De esta manera, se trata el término de error en la aproximación de la serie en forma más directa. Operando, se obtiene (14) (Daners, 2006).

\section{Solución por variables aleatorias}

Esta la publicó en The American Mathematical Monthly, en agosto del 2011, el Italiano Luigi Pace.

Comienza por tomar el cociente de dos variable aleatorias $\mathrm{Y}=\mathrm{x}_{1} / \mathrm{x}_{2}$, suponiendo que $\mathrm{x}_{1}, \mathrm{x}_{2}$, son independientes e idénticamente distribuidas (i.d.) con distribución Half-Cauchy. 
Luego, se busca la $P(0 \leq Y \leq 1)$ (función de densidad de probabilidad) mediante inspección y la función densidad de probabilidad conjunta. Esta fracción se encuentra en la serie, por lo que al integrar término a término:

$$
P\left(a \leq X_{i} \leq b\right)=\int_{a}^{b} P_{x i}(t) d t \text { (35) }
$$

La función de densidad de la función Y es:

$$
P(u)=\int_{a}^{\infty} t P_{x 1}(t u) P_{x 2}(t) d t
$$

La función de densidad conjunta es:

$$
P(a \leq Y \leq b)=\int_{0}^{\infty} \int_{a t_{1}}^{b t_{2}} P_{x 1}\left(t_{1}\right) P_{x 2}\left(t_{2}\right) d t_{1} d t_{2}
$$

Luego, al asignar la distribución Half-Cauchy a $x_{1}, x_{2}$ respectivamente:

$$
P_{x 1}(t)=\frac{2}{\pi(1+t)^{2}}
$$

$Y$ al usar la fórmula para $\mathrm{P}(\mathrm{u})$ e integrando el resultado de o a 1, se obtiene un problema equivalente al PB (Pace, 2011):

$$
\int_{0}^{1} \frac{\ln (u)}{u^{2}-1}=\frac{\pi^{2}}{8} \text { (39) }
$$

\section{Solución por discretización de ecuaciones diferenciales}

Otra demostración al problema la publicó en marzo del 2016 Mats Vermeeren, del Institut für Mathematik de la Universidad Técnica de Berlín. Allí se plantea la solución al problema mediante la discretización de ecuaciones diferenciales.

La solución de la ecuación en diferencias $x_{j+1}-2 x_{j}+x_{j-1}=-h^{2} x_{j}$, se obtiene como una serie de potencias; las soluciones de esta interpolan exactamente la discretización. Luego, por medio de la comparación de Stormer-Verlet y la discretización del oscilador armónico con su ecuación modificada, se obtiene la expansión:

$$
\left(\arcsin \frac{h}{2}\right)^{2}=\frac{1}{2} \sum_{k=1}^{\infty} \frac{(k-1) !^{2}}{(2 k) !} h^{2 k}
$$

Al final:

$$
\sum_{k=1}^{\infty} \frac{2(k-1) !^{2}}{(2 k) !}=\frac{\pi^{2}}{9}(41)
$$

Al realizar un cálculo elemental, se llega a la solución:

$$
3 \sum_{k=1}^{\infty} \frac{(k-1) !^{2}}{(2 k) !}=\frac{\pi^{2}}{6}
$$

Luego de algunas operaciones, se obtiene (14) (Vermeeren, 2016).

\section{RESULTADOS Y DISCUSIÓN}

Luego de este recorrido histórico que llega al 2016, se plantea una solución sencilla pero alternativa al PB.

Dado el espacio vectorial $V=L_{2}[-\pi, \pi]$ y una base $\beta$ ortogonal para $\mathrm{W}$ subespacio de $\mathrm{V}$, el teorema de la "descomposición ortogonal" indica que todo $f$ de $V$ puede escribirse de manera única como:

$$
f=\hat{f}+h
$$

Con $\hat{\mathrm{f}}$ en $\mathrm{W}$ y $\mathrm{h}$ en $\mathrm{W}^{\perp}$. Si $\beta$ es una base ortogonal de $\mathrm{W}$, entonces:

$$
\hat{f}=\frac{\left\langle f, g_{1}\right\rangle}{\left\langle g_{1}, g_{1}\right\rangle} g_{1}+\cdots \frac{\left\langle f, g_{p}\right\rangle}{\left\langle g_{p}, g_{p}\right\rangle} g_{p} ; h=f-\hat{f}
$$

A $\hat{f}$ se le conoce como proy $_{w}$ f: la proyección ortogonal de $f$ sobre el subespacio $W$.

La interpretación geométrica de la proyección ortogonal de un vector sobre un subespacio es sencilla: cada uno de los términos en de la proyección ortogonal de $f$ sobre el eje de una base ortogonal de W. Al sumar todas esas proyecciones, se obtiene un vector de $W$ que es la proyección ortogonal de $f$ sobre W. Es interesante hacer notar que la proyección ortogonal es única y siempre la misma, independiente de la base ortogonal para W que se esté usando.

Asimismo, se conoce que, dado un vector s en un espacio con producto interno, y dado un subespacio $\mathrm{W}$ de dimensión finita, se puede aplicar el teorema de Pitágoras a la descomposición ortogonal:

$$
\|s\|^{2}=\left\|\operatorname{proy}_{w} s\right\|^{2}+\left\|s-\operatorname{proy}_{w} s\right\|^{2}(45)
$$

Esto implica que la norma de la proyección ortogonal \|pro$y_{w} s \|$ siempre es menor o igual que la norma del vector $\|s\|$.

Lo anterior, junto con las desigualdades de Cauchy y la Triangular; las ecuaciones (46) y (47), respectivamente:

$$
\begin{gathered}
|\langle u, v\rangle| \leq\|u\|\|v\| \quad(46) \\
|\langle u+v\rangle| \leq\|u\|+\|v\| \quad(47)
\end{gathered}
$$

$\forall f, g \in L_{2}[-\pi, \pi]$, conducen al resultado central que se propone para solucionar el PB.

Teorema 1: Sea $\mathrm{W}$ un subespacio de un espacio vectorial $\mathrm{V}$, $f$ un vector de $V$, entonces, la proyección ortogonal de f sobre W es el punto más cercano a $\mathrm{f}$ de $\mathrm{W}$ :

$$
\operatorname{dist}(f, \hat{f})<\operatorname{dist}(f, w) \text { ó }\|f-\hat{f}\|<\|w-f\|
$$

Para todo $\mathrm{w}$ en $\mathrm{W}$ distinto de $\hat{\mathrm{f}}$.

La interpretación de este teorema es la siguiente: si se supone que solo se dispone de los vectores del subespacio 
W para describir los vectores del espacio total $\mathrm{V}$, entonces, dado un vector general $f \in V$, el mejor vector dentro de $V$ para describir $\mathrm{f}$ es su proyección ortogonal $\hat{\mathrm{f}}$, en el sentido en que es el más cercano a $f$ de todos los que están en W. Si el vector $f$ ya estaba en $W$, su proyección ortogonal es él mismo $\hat{f}=f$. Adicionalmente, el vector $h=f-\hat{f}$ es el vector error, siendo el error la distancia $\mathrm{f}$ a $\mathrm{W}$, es decir, $\|\mathrm{h}\|=\|\mathrm{f}-\hat{\mathrm{f}}\|$.

Demostración: Si $v \in W$ es un vector cualquiera de $W$, entonces, como $\hat{f} \in W$, también $\hat{f}-v \in W$. El vector $h=f-\hat{f}$ es ortogonal a cualquier vector de W, por (44), y en particular a $\hat{f}-\mathrm{v}$. Por ello, el teorema de Pitágoras afirma que:

$$
\|f-\hat{f}\|^{2}+\|\hat{f}-v\|^{2}=\|f-v\|^{2}
$$

Es decir, la distancia (al cuadrado) de cualquier vector $v \neq \hat{\mathrm{f}}$ de W es mayor que la distancia (al cuadrado) $\|f-\hat{f}\|^{2}$ de $f$ a $\hat{f}$, lo que prueba el teorema (Bernard y Hill, 2006).

\section{Implementación de la solución al PB}

Definiéndose el producto interior con respecto a un intervalo $[-\pi, \pi]$ como (Anton, s. f.):

$$
\int_{-\pi}^{\pi}(f \cdot g) d l
$$

y la base ortogonal:

$$
\beta=\{1, \operatorname{Sin} t, \operatorname{Cos} t, \ldots, \operatorname{Sin} n t, \operatorname{Cos} n t\}
$$

La proyección de $t^{2}$ sobre gen $(\beta)=W$ :

$$
\begin{aligned}
\operatorname{proy}_{w} t^{2}=\frac{\left\langle t^{2,1}\right\rangle}{\langle 1,1\rangle} \cdot & +\frac{\left\langle t^{2}, \operatorname{Sin} t\right\rangle}{\langle\operatorname{Sin} t, \operatorname{Sin} t\rangle} \cdot \sin t \\
& +\frac{\left\langle t^{2}, \operatorname{Cos} t\right\rangle}{\langle\operatorname{Cos} t, \operatorname{Cos} t\rangle} \cdot \operatorname{Cos} t+\cdots \\
& +\frac{\left\langle t^{2}, \operatorname{Sin} n t\right\rangle}{\langle\operatorname{Sin} n t, \operatorname{Sin} n t\rangle} \cdot \operatorname{Sin} n t \\
& +\frac{\left\langle t^{2}, \operatorname{Cos} n t\right\rangle}{\langle\operatorname{Cos} n t, \operatorname{Cos} n t\rangle} \cdot \operatorname{Cos} n t
\end{aligned}
$$

Utilizando (50):

$$
\begin{aligned}
\operatorname{proy}_{w} t^{2}=\frac{\int_{-\pi}^{\pi} t^{2} d t}{\int_{-\pi}^{\pi} d t} & \cdot 1+\frac{\int_{-\pi}^{\pi} t^{2} \operatorname{Sin} t d t}{\int_{-\pi}^{\pi} \operatorname{Sin} t \operatorname{Sin} t d t} \cdot \sin t \\
& +\frac{\int_{-\pi}^{\pi} t^{2} \operatorname{Cos} t d t}{\int_{-\pi}^{\pi} \operatorname{Cos} t \operatorname{Cos} t d t} \cdot \operatorname{Cos} t+\cdots \\
& +\frac{\int_{-\pi}^{\pi} t^{2} \operatorname{Sin} n t d t}{\int_{-\pi}^{\pi} \operatorname{Sin} n t \operatorname{Sin} n t d t} \cdot \sin n t \\
& +\frac{\int_{-\pi}^{\pi} t^{2} \operatorname{Cos} n t d t}{\int_{-\pi}^{\pi} \operatorname{Cos} n t \operatorname{Cos} n t d t} \cdot \operatorname{Cos} n t
\end{aligned}
$$

Al solucionar dichas integrales por métodos elementales y evaluar en los límites de integración: $\operatorname{proy}_{w} t^{2}=\frac{\frac{2}{3} \pi^{3}}{2 \pi} \cdot 1+\frac{0}{\pi} \cdot \operatorname{Sin} t+\frac{-4 \pi}{\pi} \cdot \operatorname{Cos} t+\frac{0}{\pi}$.

$\operatorname{Sin} 2 t+\frac{\pi}{\pi} \cdot \operatorname{Cos} 2 t+\cdots$

Simplificando:

$$
\operatorname{proy}_{w} t^{2}=\frac{\pi^{2}}{3}-4 \cdot \operatorname{Cos} t+\operatorname{Cos} 2 t+\cdots
$$

Así, la mejor aproximación de al es:

$$
\operatorname{proy}_{w} t^{2}=\frac{\pi^{2}}{3}+4 \sum_{k=1}^{n} \frac{(-1)^{k} \operatorname{Cos} k t}{k^{2}}
$$

En el caso en que se utilicen todas las funciones de $\beta$, al implementar el paso al infinito:

$$
t^{2}=\frac{\pi^{2}}{3}+4 \sum_{k=1}^{\infty} \frac{(-1)^{k} \operatorname{Cos} k t}{k^{2}}
$$

Evaluando t en un punto específico :

$$
\pi^{2}=\frac{\pi^{2}}{3}+4 \sum_{k=1}^{\infty} \frac{(-1)^{k} \operatorname{Cos} k \pi}{k^{2}}
$$

La función $\operatorname{Cos} k \pi$ con $k \in N$, siempre será igual a $(-1)^{k}$, por tanto:

$$
\pi^{2}=\frac{\pi^{2}}{3}+4 \sum_{k=1}^{\infty} \frac{(-1)^{k}(-1)^{k}}{k^{2}} \text { (59) }
$$

Simplificando y despejando el sumatorio:

$$
\frac{\pi^{2}-\frac{\pi^{2}}{3}}{4}=\sum_{k=1}^{\infty} \frac{1}{k^{2}}
$$

Operando los términos a la izquierda de la igualdad, llegamos a (14).

Las (figuras 1 a 3) muestran gráficamente los resultados. 


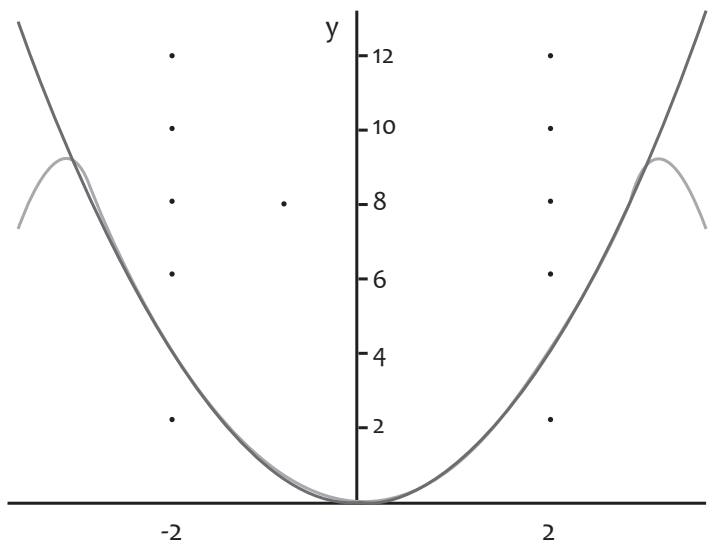

a)

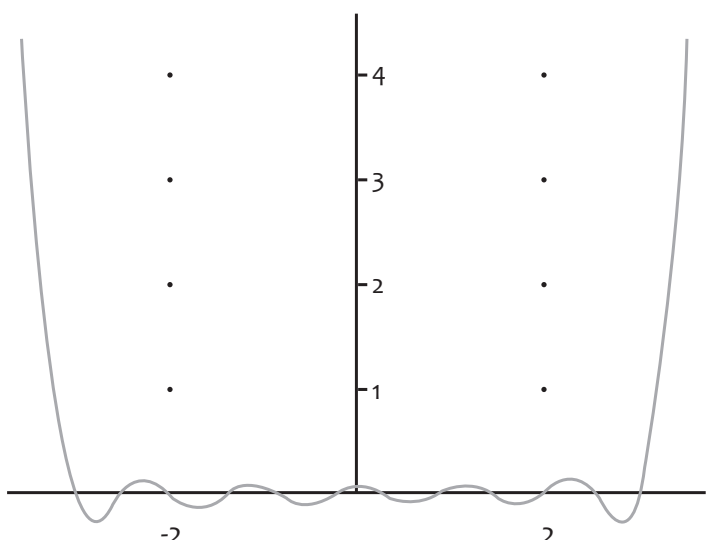

b)

Figura 1. a) Aproximación a la función por medio de proyecciones al tomar tres funciones de w; b) distancia entre y proy $_{w} t^{2}$.

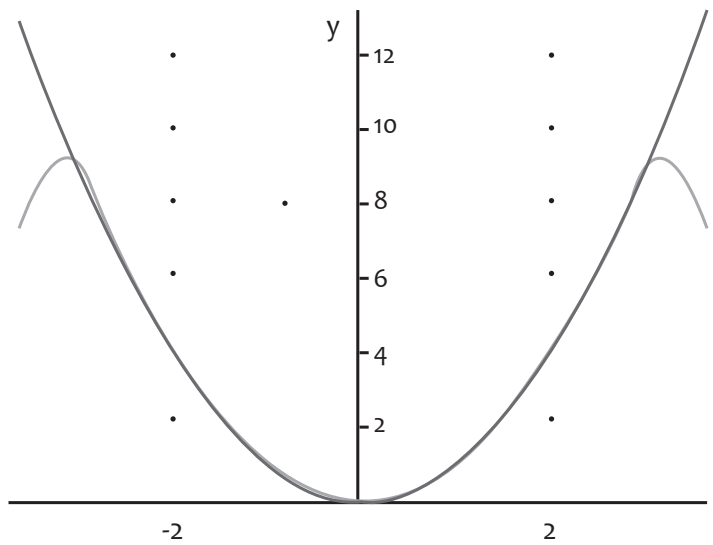

a)

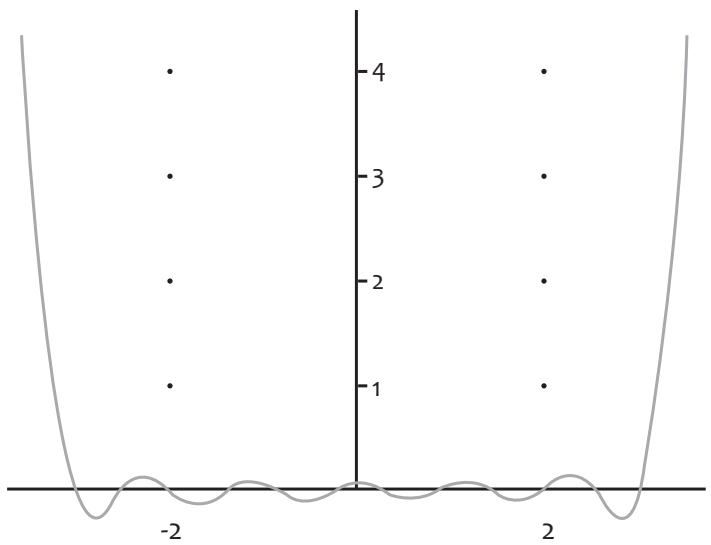

b)

Figura 2. a) Aproximación a la función $t^{2}$ por medio de proyecciones al tomar cinco funciones de w; b) distancia entre $t^{2}$ y $p r o y_{w} t^{2}$.

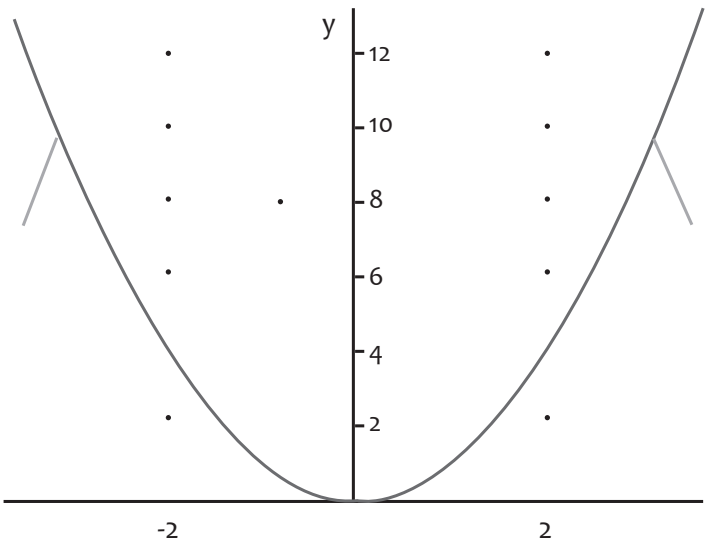

a)

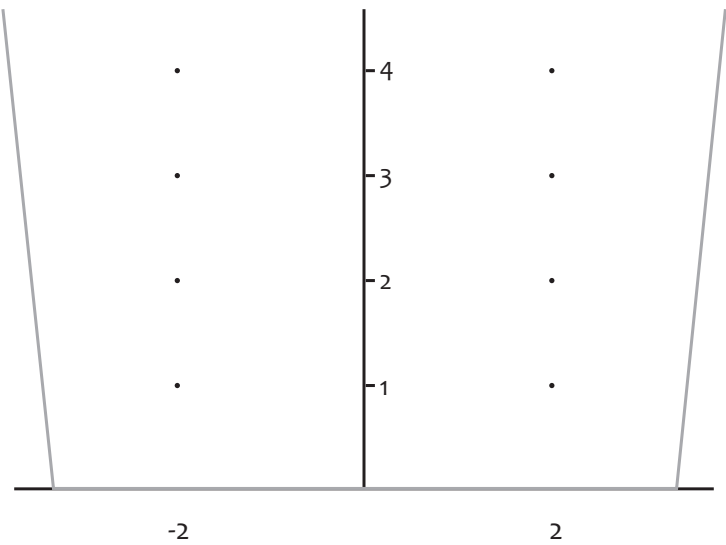

b)

Figura 3. a) Aproximación a la función por medio de proyecciones al tomar 100 funciones de w; b) distancia entre $t^{2}$ y proy $_{w} t^{2}$. 


\section{Perspectivas de la solución}

En el contexto de la solución hallada, la función Zeta de Riemann:

$$
\zeta(s)=\sum_{n=1}^{\infty} \frac{1}{n^{s}}
$$

Se denomina así en honor a Bernhard Riemann (18261866), por su investigación sobre la distribución de los números primos; conduce al PB cuando se evalúa la función Zeta en $\operatorname{dos}(2)$ :

$$
\zeta(2)=\sum_{n=1}^{\infty} \frac{1}{n^{2}}
$$

Es decir, del resultado hallado se encuentra que el valor de $\zeta(2)$ precisamente es el PB.

Como perspectiva, se puede considerar el PB desde el punto de vista de la transformación integral de Mellin, usada comúnmente en teoría de números y teoría de series asintóticas; con $f\left(e^{-t}\right)$ continua por tramos y de orden exponencial, $s$ es una variable en general compleja:

$$
M\{f(x)\}(s)=F(s)=\int_{0}^{\infty} f(x) x^{s-1} d x
$$
s. f.):

Si se parte de la función Gamma de Euler (Gadella y Nieto,

$$
\Gamma(s)=\int_{0}^{\infty} t^{s-1} e^{-t} d t \quad(64)
$$

$\mathrm{Y}$ al realizar la sustitución $\mathrm{t}=\mathrm{nx} \rightarrow \mathrm{dt}=\mathrm{ndx}, \mathrm{y}$ operando:

$$
\Gamma(s)=\int_{0}^{\infty} x^{s-1} n^{s} e^{-n x} d x
$$

Pasando a dividir:

$$
\frac{\Gamma(s)}{n^{s}}=\int_{0}^{\infty} x^{s-1} e^{-n x} d x
$$

Realizando un paso de discretización con respecto a n:

$$
\sum_{n=1}^{\infty} \frac{\Gamma(s)}{n^{s}}=\sum_{n=1}^{\infty} \int_{0}^{\infty} x^{s-1} e^{-n x} d x
$$

Al aprovechar el intercambio de operadores:

$$
\Gamma(s) \sum_{n=1}^{\infty} \frac{1}{n^{s}}=\int_{0}^{\infty} x^{s-1} \sum_{n=1}^{\infty} e^{-n x} d x
$$

$\mathrm{Y}$ al considerar la parte derecha de la igualdad como una serie geométrica, se halla que:

$$
\Gamma(s) \sum_{n=1}^{\infty} \frac{1}{n^{s}}=\int_{0}^{\infty} \frac{x^{s-1}}{e^{x}-1} d x(69)
$$

Cuando $\operatorname{Re}(s)>1$; por (59):

$$
\Gamma(s) \zeta(s)=\int_{0}^{\infty} \frac{x^{s-1}}{e^{x}-1} d x \quad(70)
$$

Donde la parte derecha es precisamente la Transformada de Mellin de $f(x)=\frac{1}{e^{x}-1}$ (Gaona, 1996).

Evaluando la función en $s=2$ :

$$
\Gamma(2) \zeta(2)=\int_{0}^{\infty} \frac{x^{2-1}}{e^{x}-1} d x \quad(71)
$$

De esta manera, se relaciona el PB con las transformaciones integrales.

Como una aplicación de lo expuesto se implementarán cálculos de $\pi$ con fracciones que lo han representado, dadas por matemáticos a través de la historia.

Así, por ejemplo, Claudio Ptolomeo (85-165 d. C.) obtuvo, al usar cuerdas de un círculo y un polígono inscrito de 360 lados, la aproximación $\pi=\frac{377}{120}$, utilizando $\sqrt{3}=\operatorname{Tan} 60^{\circ}$. Zu Chongzhi (429-500 d. c.), matemático chino y astrónomo, proporcionó dos aproximaciones del número $\pi$, y sostuvo el record de la más precisa por un periodo de novecientos años. Estas son: y $\frac{355}{113}$ y $\frac{22}{7}$.

Se han encontrado en Charlton (2001) fracciones mediante la utilización de un software diseñado en $C$, que aproximan a :

$$
\begin{aligned}
& 3 / 1=3.0000000000000000 \\
& 22 \text { ク } 3.1428571428571428 \\
& 28992=3.1413043478260869 \\
& 377 / 120=3.1416666666666667 \\
& 355 / 113=3.1415929203539825 \\
& 59973 / 19090=3.1415924567836564 \\
& 69913 / 22254=3.1415925226925498 \\
& 7985325418=3.1415925721929341 \\
& 8979328582=3.1415926107340284 \\
& 9973331746=3.1415926415926414 \\
& 10434833215=3.1415926539214212 \\
& 20834166317=3.1415926534674368 \\
& 312689 / 99532=3.1415926536189365 \\
& 833719265381=3.1415926535810779 \\
& 1146408364913=3.1415926535914038 \\
& 3126535 / 995207=3.1415926535886505 \\
& 4272943 / 1360120=3.1415926535893890 \\
& 541935 \sqrt{17} 25033=3.1415926535898153 \\
& 4762775 \sqrt{ } 15160384=3.1415926535897771 \\
& 58466453 / 18610450=3.1415926535897842 \\
& 6930515522060516=3.1415926535897891 \\
& 74724506 / 23785549=3.1415926535897909 \\
& 80143857 / 25510582=3.1415926535897927 \\
& 245850922 / 78256779=3.1415926535897931
\end{aligned}
$$


Si se utiliza la solución del PB aquí presentada, al despejar se tiene:

$$
\pi=\sqrt{6 \sum_{k=1}^{\infty} \frac{(-1)^{k} \cos k \pi}{k^{2}}}
$$

Al tomar $\mathrm{n}$ como límite superior finito para efectos computacionales, se tiene que:

$$
\pi=\sqrt{6 \sum_{k=1}^{n} \frac{(-1)^{k} \cos k \pi}{k^{2}}}
$$

Por medio del Ti-Nspire CX CAS Students Software se aproximan valores al número al realizar variaciones de $\mathrm{n}$ y comparar el valor obtenido con algunas fracciones que se aproximan a este. A fin de mostrar el margen de error, se usa con la fórmula (Posadas, s. f.):

$$
\%|e|=\frac{V_{\text {práctico }}-V_{\text {teórico }}}{V_{\text {práctico }}} \cdot 100(74)
$$

Como indica la (tabla 1) se observa que la solución propuesta en (73) es una opción plausible para calcular aproximaciones de $\pi$ con un porcentaje de error muy bajo, al

\begin{tabular}{|c|c|c|c|c|c|c|c|c|c|}
\hline VALOR K & VALOR HALLADO & & & & & & & VALOR DE & \\
\hline & 2.449489742783 & 3.1428571 & 22.1 & 3.1413043 & 22 & 3.1415929 & 22 & 3.1415926535 & 22 \\
\hline \multirow[t]{8}{*}{10} & 3.04936163598 & 3.1428571 & 2.975 & 3.1413043 & 2.92 & 3.1415929 & 2.94 & 3.1415926535 & 2.94 \\
\hline & 3.132076531809 & 3.1428571 & 0.343 & 3.1413043 & 0.294 & 3.1415929 & 0.203 & 3.1415926535 & 0.303 \\
\hline & 3.140638056205 & 3.1428571 & 0.071 & 3.1413043 & 0.0212 & 3.1415929 & 0.0304 & 3.1415926535 & 0.0304 \\
\hline & 3.141497163951 & 3.1428571 & 0.0433 & 3.1413043 & 0.0061 & 3.1415929 & 0.0031 & 3.1415926535 & 0.0030 \\
\hline & 3.141583104326 & 3.1428571 & 0.0405 & 3.1413043 & 0.0089 & 3.1415929 & 0.0003 & 3.1415926535 & 0.0003 \\
\hline & 3.141591698458 & 3.1428571 & 0.0403 & 3.1413043 & 0.0091 & 3.1415929 & & 3.1415926535 & \\
\hline & 3.14159247092 & 3.1428571 & 0.0402 & 3.1413043 & 0.0092 & 3.1415929 & & 3.1415926535 & \\
\hline & 3.141592470921 & 3.1428571 & 0.0402 & 3.1413043 & 0.0092 & 3.1415929 & & 3.1415926535 & \\
\hline
\end{tabular}
compararlas con las fracciones dadas en Charlton (s. f.).

Tabla 1. Aproximaciones de $\pi$.

\section{CONCLUSIONES}

En el presente artículo se realiza una revisión histórica acerca de $\sum_{K=1}^{\infty} \frac{1}{K^{2}}$, denominado "Problema de Basilea". Se destaca su aparición recurrente por 372 años desde diferentes ramas de las matemáticas: series, integrales, teoría de números, variables aleatorias, discretización de ecuaciones diferenciales relacionadas con osciladores armónicos, y la solución aquí propuesta, relacionada con la teoría de los espacios vectoriales.

Debido a la variedad de las soluciones, puede involucrarse como un tema didáctico en la enseñanza y el aprendizaje de las matemáticas. Cabe aclarar que, aún hoy, se continúa con la recopilación de la obra completa de Leonhard Euler (Mireille, 2014).

Del presente documento también se concluye que es posible establecerse una actividad aplicada de simulación al utilizar un asistente matemático (CAS, por sus siglas en inglés), en el cálculo de $\pi$, al contrastar la solución planteada con números racionales. Este recurso ilustra la eficiencia de los métodos computacionales involucrados en resultados novedosos a viejos problemas matemáticos.

\section{AGRADECIMIENTOS}

Al grupo de investigación Scibas y al semillero Eureka, adscritos al Centro de Investigaciones y Desarrollo Científico de la Universidad Distrital Francisco José de Caldas (CIDC) por su asesoría y acompañamiento.

\section{REFERENCIAS}

- Aigner, M. y Ziegler, G. (2004). Proofs From the Book ( $3^{\mathrm{a}}$ ed.). Springer.

- Anton H. (1981). Elementary Linear Algebra ( $3^{\mathrm{a}}$ ed.).

- Apostol T. (1996). Una historia centenaria del teorema de los números primos.

- Bernard, K y Hill, R. (2006). Algebra lineal (8 ed.). México: Pearson, Noucaoton.

- Brendan, W. (2013). The Basel Problem.

- Bry, T. (1584). Cl. Ptolomeus Alexandrinus Mathematicus. Bibliotheca Chalcographica di Jean-Jacques Boissard.

- Charlton. (Marzo de 2001). Speed Fraction! Recuperado de bach.dynet.com/ spfren/.

- Daners D. (2006). A Short Elementary Proof of $\sum_{k=1}^{\infty} 1 / k^{2}=\pi^{2} / 6$. NSW: The University of Sydney.

- Dunham, W. (2004). Euler, el maestro de todos los matemáticos. Nivola.

- Euler L. (1748). Introductio in Analysin Infinitorum (Vol. 1).

- Gadella, M. y Nieto, L. (s. f.). Métodos matemáticos avanzados para ingeniería. Departamento de Física Teórica, Universidad de Valladolid.

- Gaona, R. (1996). La transformada de Fourier y algunas de sus aplicaciones. Departamento de Matemáticas, Universidad de Sonora.

- Handmann, J. (1756). Leonhard Euler (1707-1783). Munich: Deutsches Museum.

- Knopp, K. (1996). Weierstrass's Factor-Theorem. En Theory of Functions Parts I and II, Two Volumes Bound as One, Part II (pp. 1-7). Nueva York, Dover.

- Mireille, S. (2014). Comment utilise-t-on les travaux d.Euler aujourd.hui?

- Pace, L. (Septiembre 2011). Probabilistically proving that $\zeta(2)=\pi^{\wedge} 2 / 6$. American Mathematical Monthly.

- Pérez, A. (2007). El problema de Basilea. El año de Euler: (1707-2007). Revista SUMA, 55, 95-102. 
- Plouffe, S. (2006). Identities Inspired from Ramanujan Notebooks (Part 2).

- Posadas A. (s. f.). Determinación de errores y tratamiento de datos. Departamento de Física Aplicada, Universidad de Almería.

- Rosbach, H. (2009). Zu Chongzhi. Beijing: Beijing Ancient Observatory.

- Simmons, G. (2002). Ecuaciones diferenciales con aplicaciones y notas históricas ( $2^{a}$ ed.). McGraw-Hill.

- Staehelin. (1957). Historia de la Universidad de Basilea 1632-1818. Basilea.

- Vermeeren, M. (2016). A Dynamical Solution to the Basel Problem. Institut für Mathematik, MA 7-2, Technishe Universität Berlin. 